\title{
MAGNETIC FIELD MEASUREMENTS OF THE MAIN INJECTOR SEXTUPOLE MAGNETS
}

\author{
C. M. Bhat, B. C. Brown, D. J. Harding, \\ Si J. Fang, P. S. Martin, H. D. Glass, J. W. Sim and D. G. C. Walbridge \\ Fermi National Accelerator Laboratory* P.O. Box 500, Batavia, IL 60510
}

\section{Abstract}

The Fermilab Main Injector (MI) will accelerate (decelerate) protons and anti-protons from $8.9 \mathrm{GeV} / \mathrm{c}$ to $150 \mathrm{GeV} / \mathrm{c}$ $(150 \mathrm{GeV} / \mathrm{c}$ to $8.9 \mathrm{GeV} / \mathrm{c})$. To achieve high intensity stable beam in the MI throughout the acceleration or deceleration mode, two families of chromaticity compensation sextupole magnets are planned. The operating schemes for the chromaticity compensation systems will be different for the two modes because of the hysteresis of the sextupole magnets and the changed sign of the sextupole field component from beam tube eddy currents in the dipoles. Hence we have carried out detailed magnetic field measurements on some sextupole magnets. Here we present the results of the measurements and their implications on the acceleration and the deceleration cycles of the MI.

\section{INTRODUCTION}

High quality performance of the Main Injector (MI) is important for the success of high energy $\mathrm{p} \overline{\mathrm{p}}$ collider physics programs at the Fermilab Tevatron and for several proposed fixed target experiments using the Main Injector beam[1]. The Main Injector is primarily used to accelerate high intensity protons and anti-protons $(\overline{\mathrm{p}})$ from $8.9 \mathrm{GeV}$ to $150 \mathrm{GeV}$ for Tevatron injection and protons to $120 \mathrm{GeV}$ for $\overline{\mathrm{p}}$ production. Recently we have decided to use MI to decelerate the un-used $\overline{\mathrm{p}} \mathrm{s}$ from the Tevatron and store them in the Recycler Ring[2] at $8.9 \mathrm{GeV} / \mathrm{c}$. The mode of operation for acceleration and the deceleration of the beams will be significantly different because of the hysteresis of the magnets.

The natural chromaticity of the MI is about -33 units in both horizontal and vertical planes. Particle tracking studies carried out in the MI at $8.9 \mathrm{GeV}$ (injection energy) and at $120 \mathrm{GeV}[1]$ showed that a tune spread $\Delta \nu \leq 0.0075$ is acceptable for stable operation of the beam. The $\frac{\Delta p}{p}$ of the beam at injection from the Fermilab Booster is about $1.5 \times 10^{-3}$. Then the chromaticity requirement

$$
\Delta \nu=\chi \frac{\Delta p}{p},
$$

where $\chi$ is the chromaticity, implies that $\chi \leq 5$. Similar value of chromaticity is suggested by the head-tail instability growth rate. To achieve the required chromaticity, two sets of 54 sextupole magnets[1],[3] will be inserted in the MI lattice. Also, to avoid head-tail instability after the

\footnotetext{
* Operated by Universities Research Association, Inc. under contract No. DE-AC02-76CH03000 with the U. S. Department of Energy
}

transition energy of $20.49 \mathrm{GeV}$, a chromaticity jump is introduced. A model of chromaticity compensation scheme in the presence of beam-pipe eddy current, dipole saturation and static sextupole field have been developed[4] and subsequent improvements[5] have been made to include remnant fields of the sextupole magnets for different operating scenarios. Implementation of the fast ramps with $\dot{\gamma} \approx 267 \mathrm{sec}^{-1}$ near transition, has resulted in dominance of the eddy current contribution to sextupole components at low momenta. Some of our studies indicate that below transition the focusing set of sextupole magnets may have to run in bi-polar mode (Fig. 1(a)) i.e., at $8.9 \mathrm{GeV}$ the sextupole current will be positive and for a short time during acceleration the sextupole current need to be negative to compensate for the large positive eddy current contribution to the sextupole field (Fig. 1(b)). Hence it is essential to study the low momentum behaviour of the MI chromaticity correction system.

Figure 1 shows a comparison between sextupole strength requirements for acceleration and a deceleration cycle up to $45 \mathrm{GeV} / \mathrm{c}$. The cases shown here have slight differences in their $\dot{\gamma}$ near transition, viz., $\dot{\gamma}=267 \mathrm{sec}^{-1}$ for the acceleration cycle and $\dot{\gamma}=-250 \mathrm{sec}^{-1}$ for the deceleration cycle. However, it is evident that the eddy current contributions have opposite signs in these two cases.

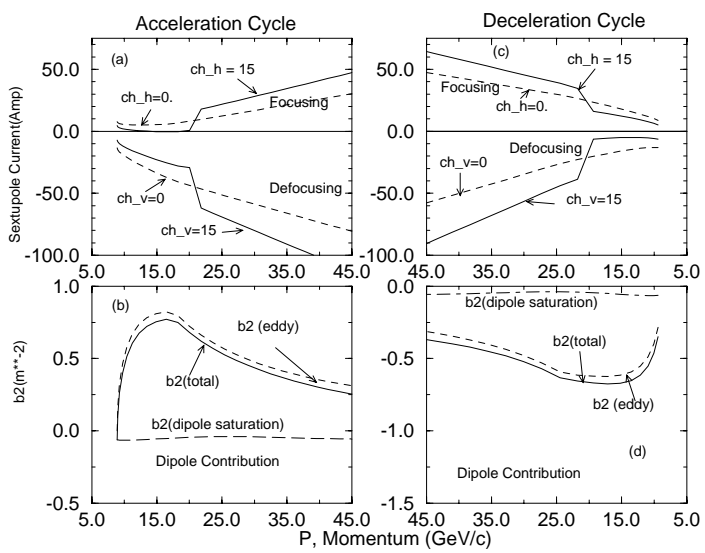

Figure 1: Main Injector sextupole current ramps as a function of the momentum for acceleration "a" and deceleration "c" cases. The sextupole contributions from the dipole magnets and contributions arising from the beam pipe eddy currents are shown in "b" and "d".

Figure 2 illustrates the sensitivity of the chromaticity as a function of the sextupole current. We notice that for focus- 
ing sets of sextupole magnets a current change of $1 \mathrm{~A}$ will introduce $\approx 4.5$ units of chromaticity change at $8.9 \mathrm{GeV}$. So, if the chromaticity needs to be controlled to better than one unit then sextupole strength error should be less than 0.2 $\mathrm{Tm} / \mathrm{m}^{2}$. This would be difficult because of the non-linear component of the sextupole field in the sextupole magnets.

To understand the low field behavior of the MI sextupole magnets extensive measurements have been undertaken at the Fermilab Magnet Test Facility (MTF). This paper presents data on the low field measurements of randomly selected sextupole magnets.

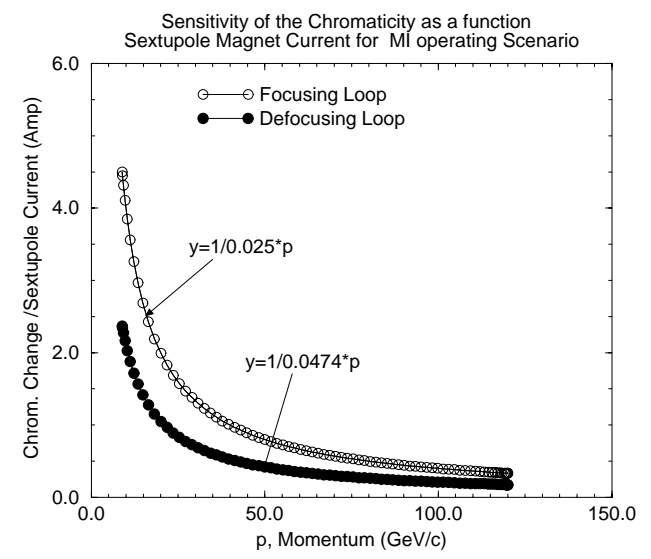

Figure 2: Sensitivity of the horizontal and vertical chromaticity as a function of the momentum.

\section{MEASUREMENTS AND RESULTS}

\subsection{Power Supply}

A special purpose power supply has been built to make detailed magnetic field measurements of the MI sextupole magnets at the MTF. The power supply is capable of providing -40 A to 350 A current with a maximum error of $100 \mathrm{~mA}$ below $10 \mathrm{~A}$ and $1 \%$ current regulation in the remaining current range. The power supply was built with two $20 \mathrm{~V} / 250$ A power supplies connected in master-slave configuration to supply forward current up to $350 \mathrm{~A}$. The reverse current is supplied by another $30 \mathrm{~V} / 65 \mathrm{~A}$ power supply with summing power diodes. The maximum voltage swing of the test power supply is $\pm 15 \mathrm{~V}$ and the maximum current slew rate is set at $400 \mathrm{~A}$ per second.

\subsection{Field Measurements}

The magnets are measured at MTF using a rotating Morgan coil with the data base-controlled software[6]. The coil is rotated at the center of the magnet at a constant current. Sextupole field measurements are performed under different conditions of Main Injector operating scenarios except for the rate of change of field. Since the dipole saturation at high field provides as high as 60 units of negative chromaticity in horizontal plane, the measurement scheme also emphasizes the high field characteristics of the dipoles. During MI operation the magnets have to be ramped to their

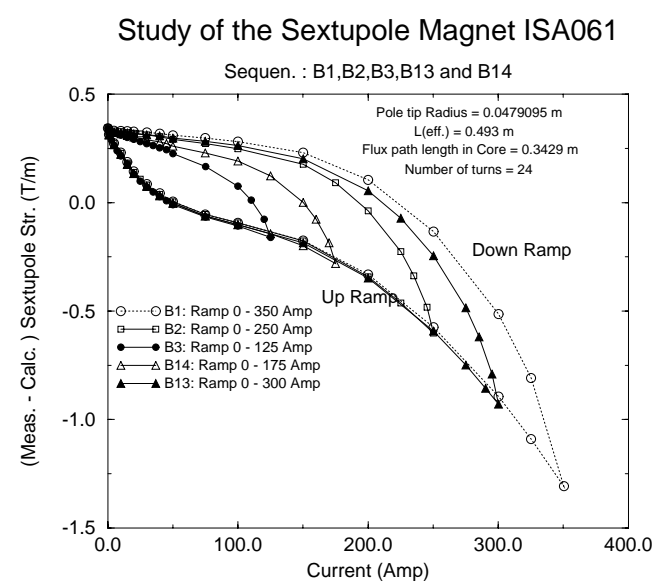

Figure 3: Remnant field as a function of sextupole current.

full strength up and down in about two to three seconds. Depending upon the details of the Fermilab accelerator "super cycle", the operating field strengths of the magnets will correspond to either $120 \mathrm{GeV}$ or $150 \mathrm{GeV}$. However, the time required for each measurement with the rotating coil system mentioned above is about $0.5 \mathrm{~min}$. Hence measurements under exact conditions are not possible. At low fields, measurements are carried out in the range of $-40 \mathrm{~A}$ to $20 \mathrm{~A}$ in steps of $1 \mathrm{~A}$. To understand the hysteresis effects at low fields we ramped the magnets to different maximum currents.

\subsection{Results of Data Analysis}

The magnetic field data analysis done here is based upon the formalism described in Refs. [7]. There are mainly two steps involved in the data analysis. In the first step, three data sets corresponding to sextupole currents below $100 \mathrm{~A}$ are used to determine the true magnetic aperture $A$ of the sextupole magnets. They are found to vary within $0.06 \%$ for the magnets studied here. In the second step, the nonlinear components of the magnetic field is determined according to,

$$
B_{\text {non-linear }}=B_{\text {meas }}-\frac{\mu_{0} 3 N L_{e f f} I}{2 A^{3}} .
$$

where $B_{\text {meas }}$ is the measured integrated sextupole strength, $N=24$ is number of coil turns driving gap, $I$ is the current, $L_{e f f}$ is the effective length of the magnet.

Some typical results of measurements are shown in Figs. 3 to 6. Figure 3 displays non-linear components of the sextupole magnets for five different maximum currents, viz., 125, 175, 250, 300 and $350 \mathrm{~A}$. In all these cases the ramps start at $0.0 \mathrm{~A}$. We find that the paths followed during up-ramp is the same within $0.025 \mathrm{Tm} / \mathrm{m}^{2}$ for all maximum current, while during down-ramps they differ significantly. The addition of the deceleration cycles of $\bar{p}$ to the MI operating scenarios suggests the necessity of use of these data (or fitted curve to these data) in the MI sextupole magnets 


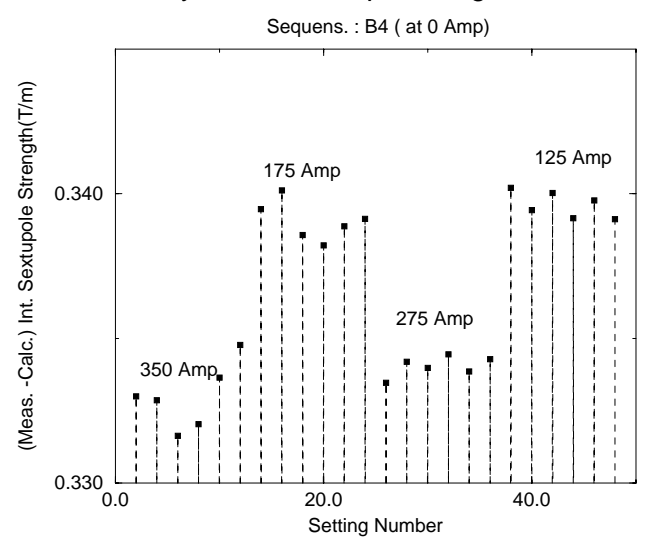

Figure 4: Remanent field for different maximum sextupole magnet current.

Study of the Sextupole Magnet ISA010

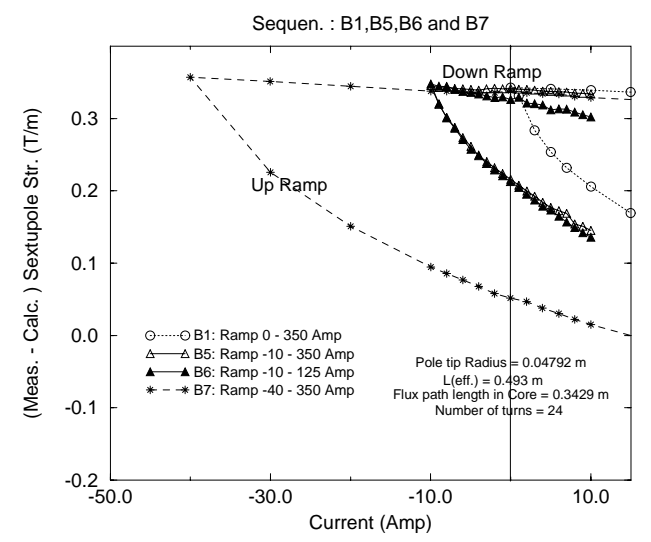

Figure 5: Remanent field for different bi-polar operation.

power supply control system[8]. In Fig. 4, the remanent field as a function of maximum ramp current is shown. The maximum deviation is about $0.005 \mathrm{Tm} / \mathrm{m}^{2}$ which will not have any noticeable effect on the chromaticity. The data taken for negative currents are shown in Fig. 5. The open triangles showing - 10 A to 350 A data and filled triangles showing - 10 A to 125 A data follow almost identical paths during up-ramp, while they follow quite different paths during down ramps. This feature is very similar to the data shown in Fig. 3 where the minimum current is $0.0 \mathrm{~A}$.

Data taken in a sequence where current is changed from (i) $10 \mathrm{~A}$ to $0 \mathrm{~A}$ to $20 \mathrm{~A}$ and, (ii) $10 \mathrm{~A}$ to $-10 \mathrm{~A}$ to $20 \mathrm{~A}$ are shown in Fig. 6. This data is very crucial in establishing the performance of the chromaticity correction in the energy range $10 \mathrm{GeV} / \mathrm{c}$ to $21 \mathrm{GeV} / \mathrm{c}$ because the sextupole strength versus momentum response curve shown in Fig. 1(a) (Focusing) has negative slope.

\section{SUMMARY}

We have carried out detailed measurements on several chromaticity compensation sextupole magnets to under-

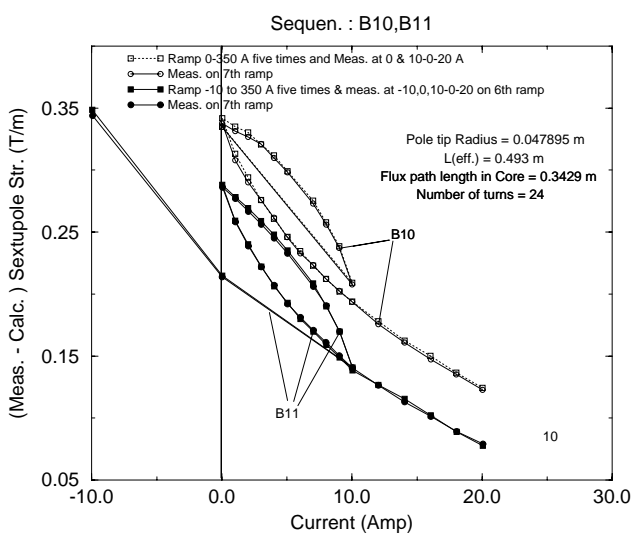

Figure 6: Remanent field for (B10) $10 \mathrm{~A}$ to $0 \mathrm{~A}$ to $20 \mathrm{~A}$ and, (B11) $10 \mathrm{~A}$ to $-10 \mathrm{~A}$ to $20 \mathrm{~A}$.

stand the non-linear behavior of these magnets at low magnetic fields. Since the chromaticity of the MI beam has to be controlled within a few units these data (or a fitted curve to these data) will be down-loaded to MI control system.

Authors would like to thank the MTF personnel for their help during the magnet measurements.

\section{REFERENCES}

[1] The Fermilab Main Injector Technical Design Handbook (1994, updated 1997) (unpublished).

[2] "Fermilab Recycler Ring Technical Design Report",edited by G. Jackson, FERMILAB-TM-1981

[3] D.J. Harding et al., IEEE Proceedings of the 1993 Particle Accelerator Conference, Washington D.C. (IEEE, New York, 1993), p. 2826

[4] S.A. Bogacz, IEEE Proceedings of the 1993 Particle Accelerator Conference, Washington D.C. (IEEE, New York, 1993), p. 77.

[5] C.M. Bhat et al., IEEE Proceedings of the 1995 Particle Accelerator Conference, Dallas, Texas (IEEE, New York, 1996), p. 1334.

[6] J.W. Sim et al., IEEE Proceedings of the 1995 Particle Accelerator Conference, Dallas, Texas (IEEE, New York, 1996), p. 2285.

[7] B.C. Brown, "Analysis of Magnet Strengths from Steel B-H Curves and Geometry" MTF-94-0078 (1997).

[8] B.C. Brown et al.,, "Design for Fermilab Main Injector Magnet Ramps Which Account for Hysteresis" this proceeding. 